\title{
Sostenibilidad y resiliencia socioecológica en el delta del Ebro*
}

\author{
Francesc Romagosa \\ Lorenzo Chelleri \\ Antonio José Trujillo Martínez \\ Françoise Breton
}

Universitat Autònoma de Barcelona. Departament de Geografia

francesc.romagosa@uab.cat

lorenzo.chelleri@uab.cat

antoniojose.trujillo@uab.cat

francoise.breton@uab.cat

\section{Resumen}

En este artículo, se presenta el delta del Ebro como un ejemplo de sistema socioecológico costero frágil que se encuentra expuesto no sólo a las consecuencias del cambio climático actual, sino también a las actuaciones humanas que amenazan a la propia sostenibilidad del delta. El artículo empieza realizando una breve presentación conceptual entorno a la resiliencia socioecológica y su relación con la sostenibilidad, en tanto que conceptos clave para interpretar la evolución y las adaptaciones recientes y futuras del territorio analizado. A continuación, se ofrece una presentación del territorio deltaico y su transformación, destacando los retos y los problemas socioecológicos a los que deberá hacer frente el delta a lo largo de las próximas décadas. Le sigue el análisis de los planes y de los instrumentos de carácter territorial y ambiental desarrollados e implementados durante las tres últimas décadas en el delta del Ebro, para finalizar con la discusión en torno a la sostenibilidad y la resiliencia futura de este socioecosistema.

Palabras clave: delta del Ebro; sistema socioecológico; sostenibilidad; resiliencia; planificación territorial y ambiental.

* Este artículo es una versión ampliada y revisada de la comunicación «Equilibrio, resiliencia y resistencia en el delta del Ebro", presentada en el I Congreso Iberoamericano de Gestión Integrada de Áreas Litorales (Cádiz, enero de 2012), y sus contenidos están basados en la investigación llevada a cabo en el proyecto WETPLAN: Servicios de ecosistemas y planeamiento adaptativo: El caso de estudio de las zonas húmedas, financiado por el Ministerio de Ciencia e Innovación (CSO 2009-08918). 
Resum. Sostenibilitat i resiliència socioecologica al delta de l'Ebre

En aquest article, es presenta el delta de l'Ebre com un exemple de sistema socioecològic costaner fràgil que es troba exposat no només a les conseqüències del canvi climàtic actual, sinó també a les actuacions humanes que amenacen la pròpia sostenibilitat del delta. L'article comença realitzant una breu presentació conceptual entorn de la resiliència socioecològica i la seva relació amb la sostenibilitat, com a conceptes clau per interpretar l'evolució i les adaptacions recents i futures del territori analitzat. A continuació, s'ofereix una presentació del territori deltaic i la transformació que ha sofert, destacant els reptes i els problemes socioecològics als quals haurà de fer front el delta al llarg de les pròximes dècades. Li segueix l'anàlisi dels plans i dels instruments de caràcter territorial i ambiental desenvolupats i implementats al delta de l'Ebre durant les tres últimes dècades, per finalitzar amb la discussió entorn de la sostenibilitat i la resiliència futura d'aquest socioecosistema.

Paraules clau: delta de l'Ebre; sistema socioecològic; sostenibilitat; resiliència; planificació territorial i ambiental.

\title{
Résumé. Durabilité et résilience socio-écologique au delta de l'Ebre
}

Cet article présente le delta de l'Ebre comme un exemple de système socio-écologique côtière fragile exposé non seulement aux conséquences du changement climatique actuel, mais aussi aux actions humaines qui menacent la viabilité même du delta. L'article commence par une brève présentation conceptuelle de la résilience socio-écologique et sa relation à la durabilité, comme les concepts clés pour interpréter l'évolution et les adaptations récentes et futures de ce territoire. Suit une introduction à la région du delta et de ses transformations qui souligne les principaux changements socio-écologiques attendus et les défis et problèmes de ceux qui y seront confrontés au cours des prochaines décennies. Finalement les plans et instruments de caractère territorial et environnemental développé et mis en œuvre au cours des trois dernières décennies dans le delta de l'Ebre sont analysés, ainsi que la durabilité et la résilience futures de ce socio-écosystème.

Mots clé: delta de l'Ebre; système socio-écologique; durabilité; résilience; environnement et aménagement du territoire.

\begin{abstract}
Sustainability and social-ecological resilience in the Ebro delta
This article presents the Ebro delta as an example of a fragile coastal social-ecological system that is exposed not only to the consequences of the current climate change, but also to human actions that threaten the sustainability of the delta itself. The article begins with a brief conceptual overview of social-ecological resilience and its relationship to sustainability as key concepts to better understand the recent and future evolution and adaptations of the delta. An introduction to the delta area and its transformation is then presented, emphasizing current anthropogenic changes and social-ecological challenges and problems that the delta will have to address in the coming decades. This is followed by an analysis of the spatial and environmental plans and instruments developed and implemented over the past three decades in the Ebro delta. The article concludes with a discussion about the future sustainability and resilience of this socio-ecological system.
\end{abstract}

Keywords: Ebro delta; social-ecological system; sustainability; resilience; environmental and spatial planning. 


\section{Sumario}

$\begin{aligned} \text { Introducción } & \begin{array}{r}\text { Planes e instrumentos como respuesta } \\ \text { a los retos de la sostenibilidad }\end{array} \\ \text { El territorio deltaico y su evolución } & \text { Adaptaciones para un delta sostenible } \\ \text { ¿Hacia un delta insostenible? } & \text { Conclusiones } \\ & \text { Referencias bibliográficas }\end{aligned}$

\section{Introducción}

En este artículo, se presenta el delta del Ebro como un ejemplo de sistema socioecológico costero frágil que se encuentra expuesto no sólo a las consecuencias del cambio climático actual, sino también a las actuaciones humanas que amenazan a la propia sostenibilidad del delta. El objetivo último del trabajo es analizar y realizar una evaluación preliminar de las distintas respuestas que la sociedad ha desarrollado durante las últimas décadas para afrontar tales desafíos, especialmente desde el punto de vista del planeamiento y la gestión ambiental y territorial. El artículo empieza realizando una breve presentación conceptual entorno a la resiliencia socioecológica y su relación con la sostenibilidad, en tanto que conceptos clave para interpretar la evolución reciente y futura del territorio analizado para, a continuación, ofrecer una presentación del territorio deltaico y su evolución hasta el momento actual, enfatizando las transformaciones antropogénicas, tanto de origen interno como externo al delta, así como los retos y los problemas socioecológicos a los que deberá hacer frente a lo largo de las próximas décadas. Le sigue el análisis de los planes y de los instrumentos de carácter territorial y ambiental desarrollados e implementados durante las tres últimas décadas en el delta del Ebro, para finalizar con la discusión en torno a la sostenibilidad y la resiliencia futura de este socioecosistema.

El contenido del artículo se basa en un análisis exhaustivo, tanto de la bibliografía disponible hasta el momento sobre la gestión territorial del delta del Ebro, como de los distintos planes e instrumentos desarrollados en este mismo ámbito. Complementariamente, se han incorporado al análisis aportaciones de los autores obtenidas a partir de las entrevistas realizadas a agentes relevantes del territorio en el marco del proyecto Wetplan entre 2008 y 2010 y de la propia observación directa y observación participante, en una metodología de investigación que combina distintas fuentes de información (Yin, 2003).

En relación con el estudio del delta del Ebro, consideramos que el enfoque integrado y territorial —-socioecosistémico- que este artículo ofrece es nuevo en la literatura científica, teniendo en cuenta que la mayor parte de los estudios publicados hasta el momento que tienen como escenario el delta del Ebro se han centrado en aspectos de carácter más específico, tales como los biofísicos (véanse, por ejemplo, entre otras referencias: Guerrero et al., 1993; Ibáñez et al., 1997; Sánchez-Arcilla et al., 1998; Cardoch y Day, 2002; Mikhailova, 2003; Curcó, 2006; Rovira e Ibáñez, 2007; Valdemoro et al., 2007). Otros 
estudios - muy pocos - ofrecen un enfoque más territorial, pero o bien no están íntegramente dedicados al delta del Ebro ni actualizados (Breton y SauriPujol, 1997), o bien sólo se centran en el análisis de una parte concreta de la gestión ambiental y territorial de la zona, como es la gestión hidrológica (Day et al., 2006; Tàbara et al., 2008) o los efectos del cambio climático (Fatoric y Chelleri, 2012). Así pues, con este artículo, pretendemos contribuir a un mejor conocimiento del delta del Ebro y sus dinámicas territoriales, subrayando la historia reciente de las políticas y las prácticas de gestión y planeamiento territorial y ambiental y las perspectivas futuras desde el punto de vista de la sostenibilidad y de la resiliencia.

\section{Sobre la resiliencia socioecológica}

Las estrategias de adaptación al cambio climático están generalmente muy asociadas al concepto de resiliencia, un concepto tan generalista que permite ser aplicado en muchas disciplinas, desde la psicología (Kaplan, 1999), hasta la ingeniería (Avallone et al., 2006), pasando por la ecología (Holling, 1973) y la antropología (Escalera y Ruiz, 2011), sólo por poner unos ejemplos. Algunas de las críticas a ese concepto vienen mayoritariamente por su borrosidad (Markusen, 1999) o por ser relativamente nuevo (Lagendijk, 2003) y, por ello, necesitar mucha más investigación y desarrollo. En cualquier caso, en este artículo, nos proponemos introducir el concepto de resiliencia socioecológica aplicado a las estrategias de adaptación —a la transformación humana y al cambio climático- adoptadas en el delta del Ebro. Desde sus primeras interpretaciones, relacionadas con la capacidad de un sistema ecológico de volver al equilibrio previo pasada una perturbación (Holling, 1973), recuperando así las funciones, la estructura y la identidad previas del sistema, la resiliencia ha desarrollado muchas más perspectivas (Walker et al., 2004) en cuanto a la capacidad del socioecosistema, no sólo de recuperación de equilibrios previos, sino también de evolución (Holling, 1996) y transformación (Folke et al., 2010). A partir de esas nuevas perspectivas, en el mundo académico no ha sido hasta muy recientemente que se ha empezado a realizar un análisis teorético transversal sobre la relación del concepto de resiliencia con las esferas de las ciencias de la sostenibilidad (Miller et al., 2010) y de la vulnerabilidad (Turner, 2010). Hasta ahora, los estudios relacionados con la adaptación (no sólo al cambio climático) estaban relacionados tanto con la vertiente de las ciencias ecológicas (Scheffer et al., 2001), como socioambientales (Folke y Berkes, 1998) o sociales (Adger, 2000), pero siempre considerando la resiliencia como una propiedad relacionada con el corto plazo, en referencia a la reacción o prevención de un impacto o una presión. Mientras Redman y Kizing (2003) debatían el peligro de vincular la resiliencia a la conservación con una visión hacia el corto plazo, la emergente preocupación por el cambio climático (IPCC, 2001) y el cambio global (Rockström et al., 2009; Reid et al., 2010) hacía evidente la necesidad de establecer un enlace entre los principios de adaptación (a los retos actuales y futuros) y la política y la gobernanza territorial (Folke et al., 2005). Es así que la resiliencia, en su definición más reciente, 
se entiende como la capacidad de adaptación y transformación de un sistema socioecológico hacia la sostenibilidad futura (Walker y Salt, 2006; Folke et al., 2010). Por consiguiente, debemos entender también la sostenibilidad de un socioecosistema como un elemento dinámico y cambiante (Escalera y Ruiz, 2011). Este enlace teórico y metodológico entre resiliencia y sostenibilidad pasa por la aplicación de estrategias adaptativas que sepan implicar una transición en los sistemas, de manera que los impactos potenciales dejen de ser una amenaza y pasen a ser futuras partes funcionales del sistema. La innovación, la transformación y la dimensión política de las adaptaciones son partes fundamentales de ese proceso de adaptación sostenible (Labin, 2005) y, por lo tanto, en nuestro estudio de caso, analizaremos los instrumentos y los planes territoriales y ambientales aplicados en el delta del Ebro para entender las dimensiones de la resiliencia socioecológica en su acepción actual.

\section{El territorio deltaico y su evolución}

El delta del Ebro, localizado al sur de Cataluña, tiene un área de $320 \mathrm{~km}^{2}$ y representa una de las principales zonas húmedas del Mediterráneo occidental, por su extensión e importancia ecológica. Como la mayoría de deltas del mundo, el del Ebro se ha desarrollado a lo largo de los últimos 5.000-7.000 años, como consecuencia de la progradación sedimentaria que tuvo lugar a partir de la última estabilización del nivel relativo del mar. Hasta el principio del siglo XX, el delta fue ganando territorio al mar, en la medida que se iban creando sucesivos lóbulos a partir del avance de distintos canales. Durante los últimos dos mil años, han existido tres lóbulos principales (Mikhailova, 2003; Curcó, 2006). El desarrollo del lóbulo meridional llegó a su máximo al fin del primer milenio. Alrededor del año 1500, el lóbulo ya había sufrido una notable regresión una vez el antiguo canal del río había sido abandonado. El lóbulo septentrional alcanzó su máximo desarrollo en 1700. A mediados del siglo XVIII, el canal actual ya era activo y favoreció el desarrollo del lóbulo central hasta el siglo XX (figura 1). Durante las últimas décadas, algunos tramos de la costa deltaica han sufrido procesos de regresión.

El delta actual constituye un territorio anfibio, dominio del mar y de la tierra a partes iguales, que presenta una combinación de distintos paisajes y ecosistemas. Como muchos espacios antropizados y desde una perspectiva socioecológica, el delta del Ebro ofrece un marcado contraste entre los ecosistemas naturales y los espacios agrícolas que, en forma de arrozales, han transformado la mayor parte de la llanura deltaica y ahora son el elemento dominante del paisaje, puesto que ocupan más de la mitad de la superficie del delta. Los arrozales se establecieron y se expandieron durante el siglo XIX sobre antiguas marismas naturales que fueron drenadas y, a su vez, colmatadas mediante la técnica denominada colmateig, que consistía en hacer llegar el sedimento del río a través de la red de canales de regadío para colmatar estas llanuras y reconvertirlas en arrozales gracias a la deposición de una capa de sedimento suficientemente gruesa. En la medida que se iban colmatando estos ambientes, 


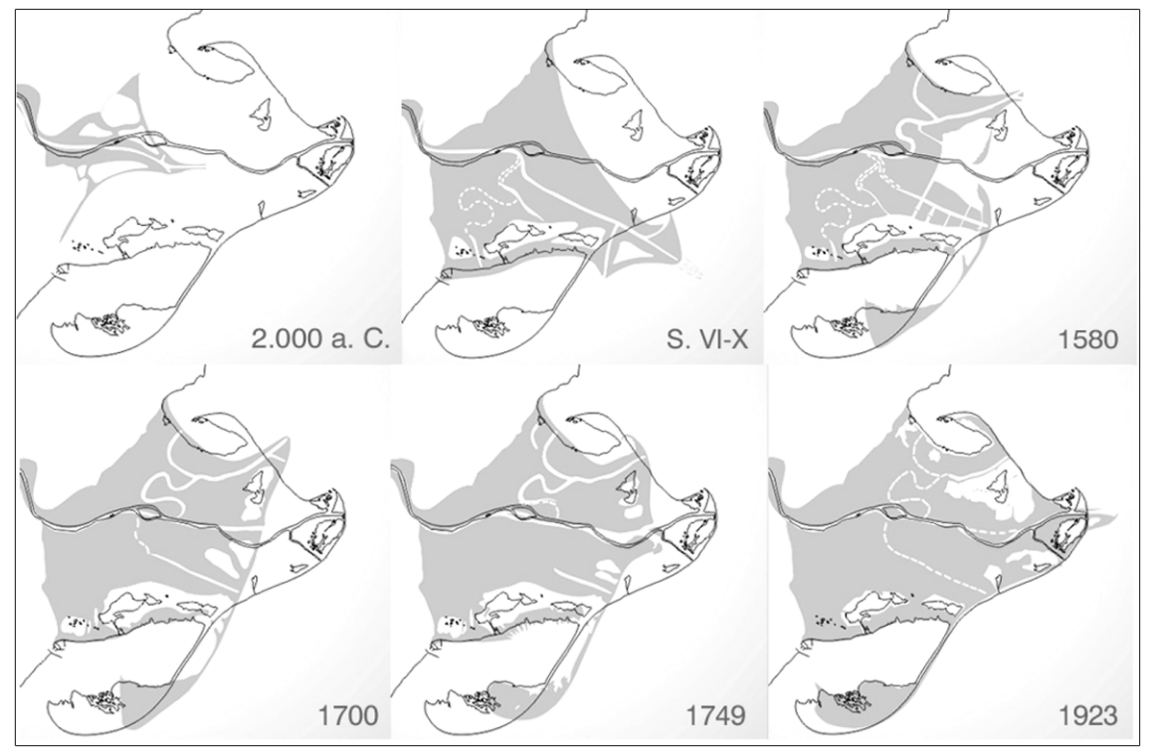

Figura 1. Evolución histórica del delta del Ebro.

Fuente: Parque Natural del Delta del Ebro (a partir de A. Canicio).

se construyó un complejo sistema de regadío mediante canales para hacer llegar los aportes del río hacia nuevas zonas del delta. La producción actual de arroz supera las 120.000 toneladas anuales, y es la tercera mayor producción dentro de la Unión Europea (Day et al., 2006). Actualmente, poco más del 20\% del delta está formado por paisajes naturales, y estos los componen mayoritariamente humedales como lagunas y marismas, pero también playas y sus sistemas dunares adyacentes. Estos espacios naturales son los que quedaron integrados dentro del Parque Natural creado en 1983. Dentro del espacio protegido, en la Punta de la Banya, se encuentran las últimas salinas operativas en el litoral catalán. Otras actividades tradicionales que se han realizado en el territorio deltaico, como la caza o la pesca, siguen realizándose, aunque de forma controlada (Parc Natural del Delta de l'Ebre, 2005). En las dos grandes bahías del delta, encontramos la moderna acuicultura, especializada en el cultivo del mejillón y, en menor medida, del ostrón y otras especies. El resto del territorio está formado por otros usos del suelo, uno de ellos es el uso urbano (el delta alberga dos ciudades en su área central, cinco en su periferia, tres pequeños núcleos rurales y dos urbanizaciones turísticas costeras. En total, suman 62.766 habitantes empadronados en 2010 (Institut d'Estadística de Catalunya, 2010).

El análisis de cambios de usos del suelo del socioecosistema del delta del Ebro realizado para el período 1990-2006 a partir de la base de datos espacial Corine, de la Agencia Europea del Medio Ambiente, nos muestra como en un período marcado por grandes transformaciones territoriales en otras zonas del 
litoral catalán, español y europeo (European Environment Agency, 2006), en el caso del delta del Ebro, estos cambios fueron, en términos generales, poco relevantes, si bien es cierto que los usos industriales y portuarios duplicaron su superficie, mientras que los usos urbanos experimentaron un ligero crecimiento, al igual que la superficie dedicada al cultivo del arroz, que se consolidó, todo ello a expensas de otros usos agrícolas y de zonas con vegetación seminatural, como pastizales o áreas periurbanas (tabla 1 y figura 2). El boom urbanístico

Tabla 1. Cambios en los usos del suelo en el socioecosistema del delta del Ebro (1990-2006)

\begin{tabular}{|c|c|c|c|c|c|c|}
\hline \multirow[b]{2}{*}{ Usos del suelo } & \multicolumn{2}{|c|}{1990} & \multicolumn{2}{|c|}{2006} & \multicolumn{2}{|c|}{$1990-2006$} \\
\hline & $\mathrm{Ha}$ & $\%$ & $\mathrm{Ha}$ & $\%$ & $\mathrm{Ha}$ & $\%$ \\
\hline Áreas urbanas & $1.463,00$ & 3,47 & $1.681,00$ & 3,99 & 218,00 & 0,52 \\
\hline Áreas industriales y portuarias & 282,00 & 0,67 & 595,00 & 1,41 & 313,00 & 0,74 \\
\hline Arrozales & $23.484,00$ & 55,70 & $23.671,00$ & 56,14 & 187,00 & 0,44 \\
\hline Otros usos agrícolas & $7.171,00$ & 17,01 & $6.619,00$ & 15,70 & $-552,00$ & $-1,31$ \\
\hline Vegetación seminatural & 779,00 & 1,85 & 614,00 & 1,46 & $-165,00$ & $-0,39$ \\
\hline Humedales, dunas y playas & $6.925,00$ & 16,42 & $6.845,00$ & 16,23 & $-80,00$ & $-0,19$ \\
\hline Cursos de agua & 877,00 & 2,08 & 880,00 & 2,09 & 3,00 & 0,01 \\
\hline Aguas marinas costeras & $1.182,00$ & 2,80 & $1.258,00$ & 2,98 & 76,00 & 0,18 \\
\hline Total $^{*}$ & 42.163 & 100 & 42.163 & 100 & & \\
\hline
\end{tabular}

* La diferencia entre la superficie analizada en esta tabla $\left(421 \mathrm{~km}^{2}\right)$ y la superficie mencionada del delta del Ebro $\left(320 \mathrm{~km}^{2}\right)$ se debe a la delimitación espacial del socioecosistema del Delta adoptada (European Environment Agency, 2010). Ésta no sólo incluye las clases principales de usos del suelo que definen el socioecosistema de zona húmeda en el Delta, sino que, posteriormente, estas clases se han ampliado usando un área de influencia de $5 \mathrm{~km}$ para incluir usos del suelo asociados a este socioecosistema. Los socioecosistemas no presentan unos límites claramente definidos y cualquier propuesta de cartografiado sigue siendo todavía una aproximación.

Fuente: elaboración propia.

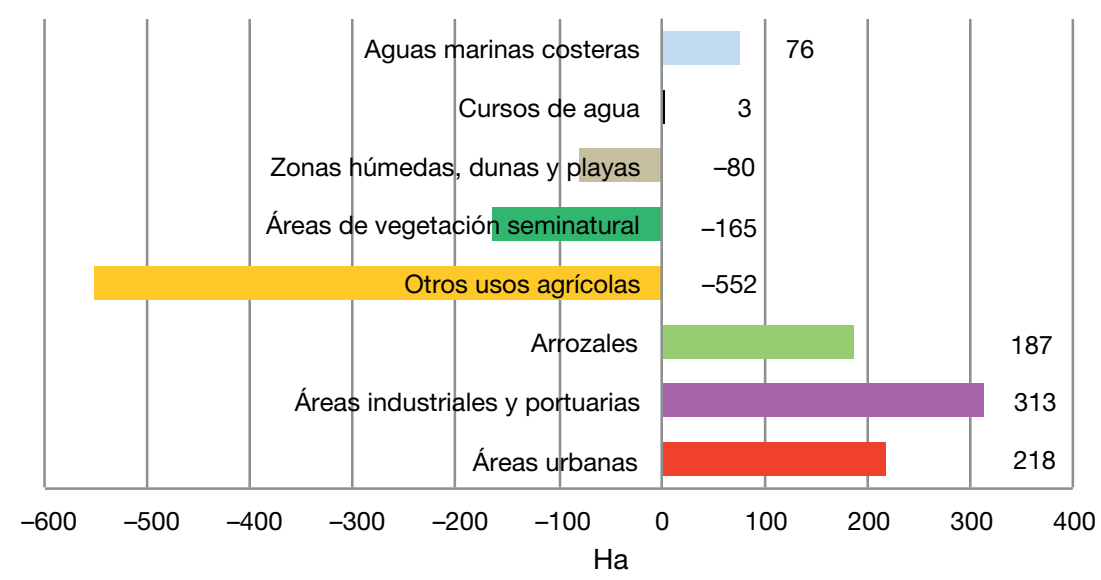

Figura 2. Cambios en los usos del suelo en el delta del Ebro (1990-2006).

Fuente: elaboración propia. 
y especulativo no tuvo tanta incidencia como en otras áreas litorales, por el hecho de que el delta ya contaba en este período con un espacio relativamente limitado para crecer, si se tiene en cuenta la superficie de espacio protegido y de producción de arroz.

\section{¿Hacia un delta insostenible?}

Los deltas son zonas húmedas, o complejos de zonas húmedas, que desempeñan un papel fundamental en la conservación de la biodiversidad, pero también en la sostenibilidad de las sociedades humanas, ya que ofrecen una larga lista de funciones o de servicios de ecosistemas (Romagosa, 2000; Millennium Ecosystem Assessment, 2005). Además, los deltas son sistemas socioecológicos con una creciente vulnerabilidad al actual cambio ambiental global. Según muchos autores, los deltas son elementos geomorfológicos frágiles que pueden sufrir cambios imprevistos y dramáticos en el equilibrio del sistema, incluso a partir de cambios modestos. Además, el desarrollo humano intensivo, el crecimiento demográfico, así como los recientes cambios globales antropogénicos, están degradando los deltas y, en muchas ocasiones, los están convirtiendo en áreas costeras altamente expuestas a riesgos. Teniendo en cuenta las tendencias actuales que incluyen el cambio climático, los cambios en la calidad y la cantidad del agua en las cuencas hidrográficas y las presiones antrópicas, muchos deltas del mundo se encuentran actualmente en peligro de colapso (Overeem y Syvitski, 2009). En este sentido, el delta del Ebro ya está afrontando varios retos y problemas socioecológicos (Day et al., 2006; Fatoric y Chelleri, 2012) a escala temporal humana que ponen en entredicho su sostenibilidad futura, entendida ésta como el mantenimiento equilibrado de sus características físicas y humanas actuales. Entre estos retos y problemas, podemos señalar:

- La disminución del caudal hídrico y una casi completa desaparición de la descarga sedimentaria del río Ebro (debida a la proliferación de embalses construidos a lo largo del siglo XX en toda la cuenca hidrológica) que lleva a la regresión costera.

- Un deterioro de la calidad de las aguas en el río, el estuario, las lagunas y las bahías, debido a un uso excesivo de productos agroquímicos (abonos, herbicidas, fitosanitarios, etc.) que circulan por la extensa red hidráulica que conforma el delta.

- Un retroceso en la pesca provocado por el deterioro de la calidad de las aguas, así como la sobreexplotación de los caladeros mediante técnicas artesanales de pesca poco sostenibles.

- Una pérdida de zonas húmedas y otros ecosistemas naturales debido a la acción humana (transformación a arrozales y zonas urbanas).

- Una subsidencia de la llanura deltaica y una falta de acreción que lleva a un hundimiento de algunas partes del delta por debajo del nivel del mar y a la consiguiente intrusión salina en los acuíferos deltaicos. 
- Unas predicciones de un ascenso acelerado del nivel del mar de entre $40 \mathrm{y}$ $100 \mathrm{~cm}$ durante el siglo XXI.

Algunos de estos problemas están provocados por las actividades humanas que se realizan en el mismo territorio, principalmente debido a patrones de ocupación y explotación humanas del medio físico. La actividad que genera un mayor impacto es la producción arrocera, ya que es la principal actividad humana en el delta, y no sólo ha transformado totalmente el paisaje, sino que también ha alterado las dinámicas hidrológica y sedimentaria (Cardoch y Day, 2002). Con el paso del tiempo, la propia actividad arrocera se ha hecho más insostenible económicamente, ya que depende en gran medida, para su supervivencia, de las subvenciones de la política agraria europea. Sin embargo, teniendo en cuenta el papel crucial que los arrozales desempeñan en la economía y en la ecología del delta (funcionando como zonas húmedas antropizadas), parece necesario mantener esta actividad productiva, introduciendo algunos cambios que la hagan más ecológicamente sostenible (por ejemplo: con la reducción de productos químicos, la regulación de los regímenes hidrológicos, el control de la salinidad del agua freática, etc.). Esto también sería beneficioso para las demás actividades económicas del delta, como la pesca o la acuicultura, que sufren impactos indirectos de la actividad arrocera intensiva.

Por lo que se refiere a la presión urbanizadora, se ha ido extendiendo y puede representar una amenaza para la conservación del patrimonio natural del delta en un futuro cercano (Tàbara et al., 2008). Esta presión se manifiesta principalmente en forma de proyectos urbanísticos (algunos de ellos fracasados y/o ilegales, como las urbanizaciones planeadas en la Punta de la Banya o en los Erms de la Tancada) o de infraestructuras (como la ampliación del puerto deportivo de Sant Carles de la Ràpita, que afecta a una parte de espacio protegido, o el proyecto descartado de aeródromo entre Amposta y Sant Carles de la Ràpita). También se refleja en la proliferación de residencias secundarias, siempre fuera de los límites del espacio protegido y de modo disperso, técnicamente definidos en muchas ocasiones como «almacenes agrícolas». Los nuevos instrumentos de planificación deberán afrontar este problema, si se quiere mantener el paisaje tradicional. La conservación de este paisaje en tanto que recurso turístico, sin dejar de ser un importante recurso económico para los productores de arroz, puede ser otro argumento potente para la protección de áreas que actualmente no gozan de protección, como es todo el sector dominado por arrozales, teniendo en cuenta que la mayor parte de turistas que van al delta del Ebro son turistas de naturaleza, interesados en la biodiversidad y en la belleza escénica del lugar.

Pero otros problemas a los que el delta debe hacer frente tienen su origen fuera de él, en la cuenca del río Ebro. Es el caso, por ejemplo, de la regresión costera, que se explica, como se ha mencionado anteriormente, por la proliferación de grandes embalses a lo largo de la cuenca hidrográfica del Ebro, que tuvo lugar durante el siglo XX y que llevó a la reducción de caudal y transporte de sedimento. Se estima que estas disminuciones fueron del orden del $50 \%$, por lo que se refiere al caudal hídrico, y del 99\%, en cuanto al volumen de sedimentos, 
entre principios y finales del siglo XX (Ibáñez et al., 1997). La progradación que había caracterizado la evolución del delta durante los últimos siglos quedó interrumpida a mediados del siglo pasado, lo cual dio paso al inicio de un período de regresión (aunque actualmente algunas áreas están progradando, la mayor parte de la línea de la costa del delta se encuentra en regresión). El estudio de la evolución de la costa deltaica muestra como este retroceso es evidente en la zona de la desembocadura del río durante la segunda mitad del siglo XX, con más de un kilómetro lineal de retroceso (Valdemoro et al., 2007). Además, la llanura deltaica se está hundiendo, porque la subsidencia — resultado de la sobreexplotación de los acuíferos y de otros procesos físicos y humanos que llevan a la compactación del paquete sedimentario-, estimada entre 1 y $6 \mathrm{~mm}$ anuales (Ministerio de Medio Ambiente, 2006), no es compensada por la entrada de nuevos sedimentos de arcillas y limos. A eso hay que añadir que las aguas de drenaje de los arrozales exportan sedimentos a una tasa equivalente a unos 0,2 mm anuales de pérdida de elevación (Ibáñez et al., 1997). En definitiva, la reducción en el aporte de arena en la línea de la costa ha conllevado la adaptación de la franja costera sin disponer de un incremento en el balance sedimentario (Sánchez-Arcilla et al., 1998). La restauración parcial de los flujos de sedimentos en el tramo final del río Ebro parecería técnicamente viable y ambientalmente deseable, tal y como se han encargado de demostrar varios estudios al respecto (Ministerio de Medio Ambiente, 2006; Rovira e Ibáñez, 2007). En este sentido, el Plan de gestión del sedimento del tramo bajo y final del río Ebro y su delta (Pla GeSt Ebre) (Agència Catalana de l'Aigua, 2007) propone la removilización de los sedimentos retenidos en embalses (sistema Mequinensa - Riba-roja - Flix) y su posterior transporte hacia el tramo final del río Ebro y la llanura deltaica. A pesar de ello, actualmente siguen desarrollándose minitrasvases en el tramo bajo y final del río y del delta (canal Segarra-Garrigues, canal Xerta-Sènia, canal de l'Aldea-Camarles, mini trasvase Camp de Tarragona, etc.), lo cual contribuye al mantenimiento del déficit sedimentario en el delta.

Finalmente, a esas prácticas locales y regionales, cabe añadir otros impactos de escala superior que afectan a la evolución del delta. Nos referimos al impacto del cambio climático, que, con la elevación del nivel del mar y la salinización de los acuíferos, está amenazando las costas bajas a escala planetaria. Por otra parte, como sucede en otros deltas, este impacto se ve agravado por la subsidencia previamente mencionada (IPPC, 2001). En el caso del delta del Ebro, una parte significativa de la llanura deltaica ya se encuentra actualmente cerca o por debajo del nivel medio del mar. Durante las próximas décadas, el principal problema geomorfológico será la pérdida de elevación del terreno en la llanura deltaica, debido a la falta de deposición sedimentaria combinada con la subsidencia y la elevación del nivel del mar (Day et al., 2006). El estudio que llevó a cabo el Taller d'Enginyeria Ambiental (2008) por encargo de la Oficina Catalana del Cambio Climático, muestra las áreas expuestas a la inundación de acuerdo con diferentes escenarios de elevación del nivel del mar: $15 \mathrm{~cm}$ en 2050 (A), $40 \mathrm{~cm}$ en 2100 (B) y $100 \mathrm{~cm}$ en 2100 (C) (figura 3). Como se puede observar, los distintos escenarios tienen en común el hecho que, si se cumplie- 

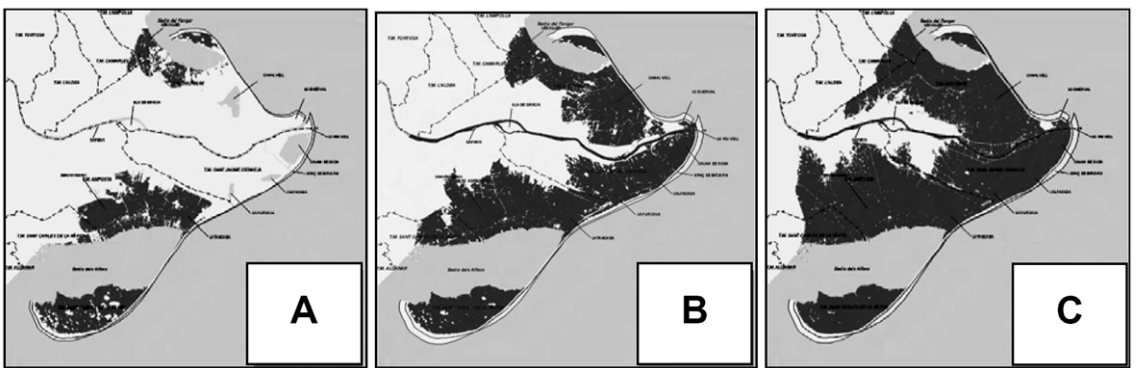

Figura 3. Escenarios de aumento del nivel del mar para el delta del Ebro. Fuente: Taller d'Enginyeria Ambiental (2008).

ran las previsiones (tanto las más pesimistas como las más optimistas), extensas áreas del delta actual quedarían inundadas. Es preciso tener en cuenta que el delta es un área extremadamente llana y este hecho lo hace especialmente vulnerable a los cambios en el nivel del mar. Además, los cambios en la dirección, la intensidad y la frecuencia de los oleajes pueden verse reflejados en cambios en el modelo de erosión, transporte y sedimentación a lo largo de la costa del delta. Sin embargo, cabe decir que estos mapas no tienen en consideración las posibles acciones de adaptación al ascenso del nivel del mar, que deberían reducir significativamente las áreas potencialmente inundables.

\section{Planes e instrumentos como respuesta a los retos de la sostenibilidad}

Durante las tres últimas décadas, han surgido distintos intentos de abordar los retos de sostenibilidad que el delta del Ebro afronta, entre ellos, la propia desaparición del delta. A continuación, analizaremos los principales planes e instrumentos que se han desarrollado en este período, que van desde la planificación y la gestión ambiental del territorio, hasta los planes territoriales de carácter general, pasando por la gestión integrada de los recursos hídricos y del territorio.

\section{Planificación y gestión ambiental}

Con el objetivo de frenar una serie de proyectos que amenazaban la supervivencia de los ecosistemas naturales del delta y, a la vez, favorecer la armonía entre los valores naturales de ese territorio y su uso por parte de los humanos en 1983, se creó el Parque Natural del Delta del Ebro (Decreto 357/1983 del Gobierno catalán). Inicialmente, el espacio protegido sólo incluía las zonas húmedas, las playas y los sistemas dunares de la franja costera del hemidelta norte, pero, en 1986, se amplió el área de protección, incluyendo la franja costera del hemidelta sur (Decreto 332/1986), fruto de un movimiento local encabezado por cazadores y los ayuntamientos de la zona, que veían en peligro 
la eliminación y transformación de la laguna del Canal Vell por parte de sus propietarios. Una vez ampliado, el parque natural abarcó 8.445 ha terrestres y 564 ha marinas. El espacio también incluyó la Reserva Natural en la Punta de la Banya, donde el nivel de protección es superior al del resto del parque. En 1992, el gobierno autonómico aprobó el Plan de Espacios de Interés Natural (PEIN) para todo el territorio catalán y esto permitió ampliar la superficie protegida del delta del Ebro. La superficie protegida total pasaría a ser de 12.378 ha terrestres y 35.647 ha en el mar. La misma superficie sería, finalmente, incorporada, en 2006, en la Red Natura 2000 (figura 4). Además, el delta del Ebro cuenta con otras figuras de protección internacional, como la Zona de Especial Protección (1987), de acuerdo con la Directiva europea de conserva-

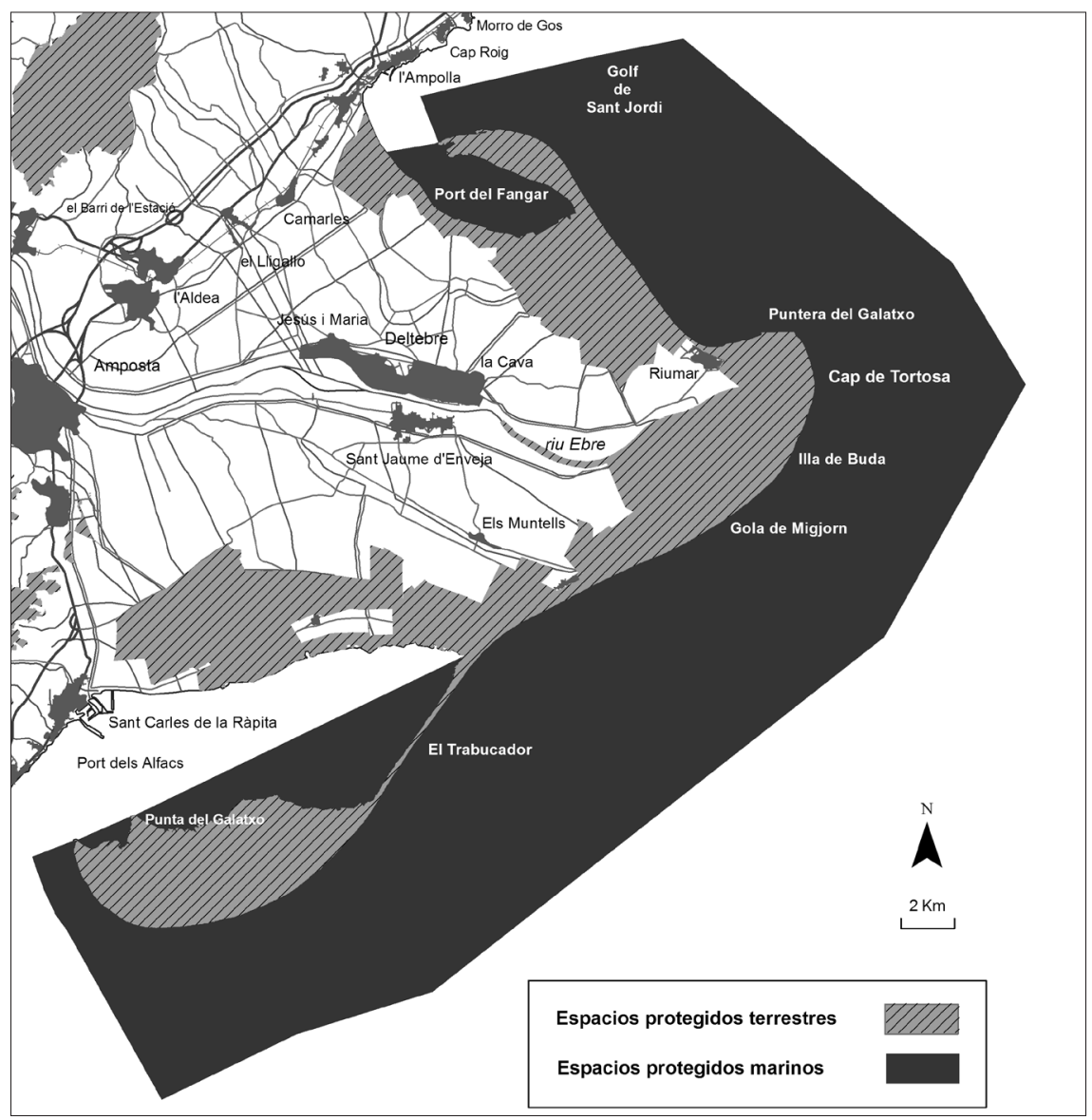

Figura 4. Espacios naturales protegidos en el delta del Ebro (Red Natura 2000). Fuente: elaboración propia. 
ción de las aves (79/408/EEC), o la resolución de 15 de marzo de 1993 para su inclusión en la lista Ramsar de humedales de importancia internacional, especialmente como hábitat de aves acuáticas.

A pesar de que ha habido varios intentos, por parte de la Administración, de ampliar la superficie protegida, integrando los arrozales en el parque natural o en la Red Natura 2000, hasta el momento los agricultores y la comunidad de regantes no han visto tal propuesta positiva para sus intereses. Sin embargo, desde un punto de vista objetivo, parecería apropiado e incluso recomendable que todo el delta tuviese una única figura de protección, puesto que ello aseguraría una planificación y una gestión ambiental integradas que, en última instancia, impulsarían la sostenibilidad global del delta (económica, social y ambiental). En cualquier caso, la figura de parque natural ha sido muy importante para la zona, porque, en muchas ocasiones, ha sido el impulsor de políticas y actuaciones que han favorecido no sólo el área protegida, sino también el conjunto del delta. Un ejemplo de este rol socioeconómico que desempeña el parque en todo el delta es el hecho de que ha sido el impulsor del proceso de convertir el lugar en un destino turístico de calidad en el contexto europeo. Ello se ha materializado en la obtención de dos distintivos de alto nivel (la Carta Europea del Turismo Sostenible, otorgada por la Federación Europarc en 2007, y el distintivo de Destino Europeo de Excelencia EDEN, otorgado por la Comisión Europea en 2009) (Parc Natural del Delta de l'Ebre, 2009). Ambos distintivos tratan al conjunto del delta (no sólo al espacio protegido) como destino turístico de naturaleza, y todo el sector turístico del área de influencia del delta debería verse beneficiado por esas iniciativas.

\section{Hacia una gestión integrada del agua y del territorio}

Durante los últimos años, el delta del Ebro ha experimentado un cambio en el concepto de gestión hídrica: el agua debería ser entendida como un recurso y un principio guía para la ordenación del territorio y de sus usos (Ministerio de Medio Ambiente, 2007). Esta idea va en la línea de la demanda creciente de la integración entre la gestión hídrica y las políticas sectoriales, uno de los conceptos clave de la Directiva Marco del Agua de la Unión Europea (2000/60/EC). La gestión hídrica, en tanto que principio estratégico para la consiguiente planificación territorial integrada, es todavía un concepto relativamente reciente en España. Una muestra de ello es la derogación del Plan Hidrológico Nacional (PHN) (RDL 2/2004 y Ley 11/2005), aprobado inicialmente en 2001 por el gobierno del Partido Popular. El nuevo gobierno socialista consideró que algunos de los principios de ese plan eran insostenibles y poco acordes a los nuevos principios de la gestión hídrica (la denominada «nueva cultura del agua») y a las políticas hídricas y ambientales europeas. Igualmente, el PHN había generado un fuerte rechazo social, no sólo en el propio delta, sino también en otros lugares de Cataluña y de España. Fue el detonante, por ejemplo, de la creación de la Plataforma en Defensa del Ebro, movimiento social que influyó decisivamente en el cambio de rumbo de las políticas hidrológicas en 
el delta del Ebro durante la primera década del siglo XXI. El PHN tenía como objetivo principal, en la parte baja del Ebro, la implementación de un trasvase de agua de un máximo de $1.050 \mathrm{hm}^{3} /$ año desde el bajo Ebro hacia el norte y el sur de la costa mediterránea. En concreto, se establecían los siguientes valores máximos anuales para ser trasvasados hacia otras cuencas: $190 \mathrm{hm}^{3}$ hacia el área de Barcelona, $315 \mathrm{hm}^{3}$ hacia Valencia, $450 \mathrm{hm}^{3}$ hacia Murcia y $95 \mathrm{hm}^{3}$ hacia Almería. Esa misma ley establecía un caudal mínimo para el Ebro de $100 \mathrm{~m}^{3} / \mathrm{s}$ $\left(3.153 \mathrm{hm}^{3} /\right.$ año$)$, y que el agua no podía ser transferida desde junio hasta septiembre. Algunas de las actuaciones inicialmente previstas por el plan incluían la construcción de más de un centenar de nuevos embalses para garantizar la distribución de las aguas trasvasadas del Ebro hacia los otros territorios y facilitar el establecimiento de más de 400.000 ha de regadío, lo cual podía generar unos impactos socioambientales negativos significativos (Day et al., 2006). En cuanto al trasvase de agua, implicaría impactos directos sobre los diversos servicios de ecosistemas identificados en el delta y cuantificados en 120 millones de euros anuales, incluyendo agricultura, pesca, acuicultura y turismo (Slootweg y Van Beukering, 2002). Con la derogación del plan, el trasvase fue abortado y el Plan Integral para la Protección del Delta del Ebro (PIPDE) inicialmente previsto también fue reformulado en 2006. Ahora, las principales actuaciones son las siguientes (Ministerio de Medio Ambiente, 2006):

— La definición de un régimen hidrológico (caudal) mínimo para mantener las funciones ecológicas básicas del delta.

- La definición de medidas para evitar la subsidencia del delta.

- La mejora de la calidad de las aguas de la agricultura, a fin de evitar la eutrofización y de promover un modelo agronómico sostenible.

- El seguimiento de las condiciones ecológicas del delta, mediante la construcción de indicadores ambientales y bases de datos fiables.

Podría ser definido como un plan de gestión integrada del agua, pero también del territorio, no cerrado en el tiempo ni en el espacio (un plan adaptativo), debido a su naturaleza de proceso continuo de seguimiento y retroalimentación de actuaciones, a la espera de la incorporación del nuevo Plan Hidrológico de la Demarcación Hidrográfica del Ebro, que también incorporará los criterios de la Directiva Marco del Agua y los principios de sostenibilidad en el uso de los recursos. Igualmente, deberá adaptarse a la planificación territorial vigente. El plan tenía la previsión de implementar actuaciones por valor de 465 millones de euros durante los tres primeros años (Ministerio de Medio Ambiente, 2006). Con la llegada de la crisis económica y financiera de 2008, el Plan ha seguido vigente, pero ha relajado la implementación de las actuaciones en el tiempo.

\section{Planificación territorial}

En cuanto a la planificación territorial, una primera constatación es el hecho que, si no tenemos en cuenta el descoordinado planeamiento urbanístico 
municipal, el delta del Ebro no tuvo ningún tipo de planificación territorial hasta la década de 1990. Hasta entonces, los asentamientos humanos habían seguido la morfología territorial del delta y sólo en tiempos recientes tuvieron que adaptarse a los cambios bruscos y a las nuevas jerarquías territoriales debidos a la aparición de nuevas infraestructuras y nuevas polaridades territoriales (especialmente, la expansión urbana). El primer plan de desarrollo del delta del Ebro (Plan Director del Delta del Ebro) empezó, en 1995, a promover el desarrollo del delta con nuevas infraestructuras, industrias y equipamientos para el turismo de masas, intentando proteger la naturaleza al mismo tiempo (Breton y Sauri-Pujol, 1997). El siguiente paso para la planificación territorial debería haber sido la integración de programas y políticas sectoriales, frenar la expansión urbana redefiniendo los usos del suelo y tener en cuenta el medio natural en el planeamiento. Pero el Plan Territorial de las Tierras del Ebro (Departament de Política Territorial i Obres Públiques, 2001), realizado y aprobado en 2001 por el Gobierno autonómico, no sólo para el territorio deltaico, sino también para todas las comarcas de su entorno, no tuvo en consideración esos aspectos. Ese plan y el mencionado Plan Hidrológico Nacional de 2001 pusieron claramente en evidencia la necesidad de reformar el marco completo de planificación para el delta del Ebro, si realmente se quería perseguir la sostenibilidad territorial y ambiental. La evolución demográfica del primer decenio del siglo XXI también contribuyó a hacer evidente esa necesidad, ya que el límite de crecimiento previsto de alcanzar para todas la Tierras del Ebro en 2016 en el plan de 2001 ya fue superado en 2007 y la mayor parte del suelo urbanizable ya había sido urbanizado durante los últimos años.

Con el nuevo Plan Integral para la Protección del Delta del Ebro (PIPDE), empezaron a incorporarse enfoques más sostenibilistas e integradores en la planificación territorial e hídrica, actuando como se ha dicho, como un auténtico plan de ordenación y planificación territorial. Lo mismo se puede afirmar del más reciente e innovador Catálogo del Paisaje de las Tierras del Ebro, aprobado en 2010, así como del nuevo Plan Territorial de las Tierras del Ebro, también aprobado definitivamente en 2010 (inicialmente, en 2009) por el Gobierno catalán y que sustituye al plan de 2001 (Departament de Política Territorial i Obres Públiques, 2009). Eso significa que este último plan será el nuevo instrumento de planificación territorial para los próximos años y que los demás instrumentos de planificación, como los planes urbanísticos, deberán seguir su marco planificador. Entre sus objetivos principales, se pueden citar:

- Favorecer la diversidad del territorio y mantener la matriz biofísica como referencia.

- Proteger las áreas naturales, el paisaje agrario y las zonas no urbanas en general como elementos básicos del planeamiento.

- Controlar la ocupación del suelo (desarrollo urbano racional, promoviendo el crecimiento urbano compacto y continuo).

- Favorecer la movilidad sostenible en la región.

- Mejorar las dinámicas energéticas y ambientales. 
Este nuevo plan representa, para el delta del Ebro, una oportunidad para contribuir a la sostenibilidad ecológica y socioeconómica, ya que se protegen todas las áreas naturales (previamente protegidas o no) y las áreas agrícolas (catalogadas como áreas agrícolas de interés socioeconómico), además, tiene en consideración las dinámicas ambientales y las amenazas del cambio climático. En cualquier caso, deberá comprobarse, a lo largo de los próximos años, si esta planificación es efectiva para hacer frente a los retos que tiene el delta del Ebro y si realmente puede asegurar un futuro sostenible para ese delta.

\section{Balance de tres décadas de planeamiento}

$\mathrm{El}$ análisis de los diferentes planes e instrumentos mencionados previamente - ya sea de acuerdo con sus contenidos y sus propuestas objetivas, ya sea de acuerdo con sus objetivos explícitos - permite constatar cómo se ha producido una evolución a lo largo de tres décadas (desde la década de 1980 hasta la actualidad) desde el punto de vista de la sostenibilidad territorial del delta del Ebro. Efectivamente, como se puede observar en la tabla 2, los planes y los instrumentos analizados aprobados en las décadas de 1980 y 1990 y primera mitad de 2000 o bien no impulsan la sostenibilidad territorial global del delta, o bien en algunos casos sólo impulsan una sostenibilidad parcial basada únicamente en aspectos medioambientales, básicamente centrados en la protección de los ecosistemas naturales, pero dejando de lado otros aspectos como la sostenibilidad económica y social del territorio. No es hasta la llegada del PIPDE, en 2006, y de los planes e instrumentos más recientes, impregnados de una nueva filosofía más sostenibilista - en coherencia con las tendencias contemporáneas en el ámbito del planeamiento territorial en España y Europa-, cuando aparecen enfoques de carácter más integrado que persiguen no sólo la conservación y la protección de los ecosistemas naturales, sino también el desarrollo económico y social equilibrado del espacio deltaico y su área de influencia. Aun así, no es evidente que estos instrumentos tengan suficiente fuerza como para hacer frente a los grandes retos que tiene planteado el delta a medio y largo plazo. Por solo poner un ejemplo, nada se dice, en ninguno de los documentos analizados, sobre qué estrategia deberán seguir ante la subida del nivel del mar las zonas urbanizadas del frente costero deltaico, como Riumar o Els Eucaliptus.

\section{Adaptaciones para un delta sostenible}

Como se ha analizado en la sección anterior, el delta del Ebro es un territorio que se ha caracterizado por una falta de atención por parte de la planificación durante muchas décadas, y que sólo recientemente ha desarrollado algunos instrumentos de ordenación territorial centrados en proteger, valorar y recuperar las peculiaridades del delta. Los estudios de cambios de usos del suelo evidencian que no ha habido cambios sustanciales en las dinámicas socioecosistémicas recientes del delta, y el cultivo del arroz sigue siendo el principal motor de 
Tabla 2. Planes e instrumentos territoriales y ambientales en el delta del Ebro

\begin{tabular}{|c|c|c|c|c|c|}
\hline Plan o instrumento & Año & $\begin{array}{l}\text { Institución } \\
\text { impulsora }\end{array}$ & Aspectos principales & Territorio cubierto & $\begin{array}{l}\text { ¿Impulsa la } \\
\text { sostenibilidad? }\end{array}$ \\
\hline $\begin{array}{l}\text { Decreto de creación } \\
\text { del Parque Natural } \\
\text { del Delta del Ebro. }\end{array}$ & 1983 & $\begin{array}{l}\text { Generalitat } \\
\text { de Catalunya. }\end{array}$ & $\begin{array}{l}\text { Protección } \\
\text { de ecosistemas } \\
\text { naturales. }\end{array}$ & $\begin{array}{l}\text { Franja costera del Delta } \\
8.445 \text { ha (terrestres) y } \\
564 \text { ha (marítimas). }\end{array}$ & $\begin{array}{l}\text { Parcialmente } \\
\text { (ambiental). }\end{array}$ \\
\hline Ley de costas. & 1988 & Gobierno español. & $\begin{array}{l}\text { Regulación de } \\
\text { la franja costera. }\end{array}$ & $\begin{array}{l}\text { Zona costera (sólo playas, } \\
\text { sistemas dunares } \\
\text { y zonas húmedas) y mar. }\end{array}$ & $\begin{array}{l}\text { Parcialmente } \\
\text { (ambiental). }\end{array}$ \\
\hline $\begin{array}{l}\text { Plan de Espacios } \\
\text { de Interés Natural } \\
\text { (PEIN). }\end{array}$ & 1992 & $\begin{array}{l}\text { Generalitat } \\
\text { de Catalunya. }\end{array}$ & $\begin{array}{l}\text { Protección } \\
\text { de ecosistemas } \\
\text { naturales. }\end{array}$ & $\begin{array}{l}\text { Franja costera del Delta } \\
12.738 \text { ha (terrestres) } \\
\text { y } 35.647 \text { ha (marítimas). }\end{array}$ & $\begin{array}{l}\text { Parcialmente } \\
\text { (ambiental). }\end{array}$ \\
\hline $\begin{array}{l}\text { Plan de Desarrollo } \\
\text { del Delta del Ebro. }\end{array}$ & 1995 & $\begin{array}{l}\text { Municipios } \\
\text { y Generalitat } \\
\text { de Catalunya. }\end{array}$ & $\begin{array}{l}\text { Desarrollo urbano y } \\
\text { de infraestructuras. }\end{array}$ & $\begin{array}{l}320 \mathrm{~km}^{2} \text { (toda la llanura } \\
\text { deltaica). }\end{array}$ & No. \\
\hline $\begin{array}{l}\text { Plan Territorial de } \\
\text { las Tierras del Ebro. }\end{array}$ & 2001 & $\begin{array}{l}\text { Generalitat } \\
\text { de Catalunya. }\end{array}$ & Plan territorial general. & $\begin{array}{l}320 \mathrm{~km}^{2} \text { (toda la llanura } \\
\text { deltaica) + comarcas } \\
\text { adyacentes. }\end{array}$ & No. \\
\hline $\begin{array}{l}\text { Plan Hidrológico } \\
\text { Nacional. }\end{array}$ & $\begin{array}{l}2001- \\
2005\end{array}$ & Gobierno español. & Gestión hidrológica. & $\begin{array}{l}320 \mathrm{~km}^{2} \text { (toda la llanura } \\
\text { deltaica) + otras regiones. }\end{array}$ & No. \\
\hline $\begin{array}{l}\text { Plan Director } \\
\text { Urbanístico del Sistema } \\
\text { Costero (PDUSC). }\end{array}$ & 2004 & $\begin{array}{l}\text { Generalitat } \\
\text { de Catalunya. }\end{array}$ & $\begin{array}{l}\text { Regulación } \\
\text { del desarrollo } \\
\text { urbanístico. }\end{array}$ & $\begin{array}{l}\text { Franja costera } \\
1.974 \text { ha. }\end{array}$ & $\begin{array}{l}\text { Parcialmente } \\
\text { (ambiental). }\end{array}$ \\
\hline $\begin{array}{l}\text { Plan Integrado para } \\
\text { la Protección del Delta } \\
\text { del Ebro (PIPDE). }\end{array}$ & 2006 & Gobierno español. & $\begin{array}{l}\text { Gestión hidrológica } \\
\text { y ambiental. }\end{array}$ & $\begin{array}{l}320 \text { km² } \\
\text { (toda la llanura deltaica). }\end{array}$ & Sí. \\
\hline Red Natura 2000. & 2006 & $\begin{array}{l}\text { Generalitat } \\
\text { de Catalunya } \\
\text { y Unión Europea. }\end{array}$ & $\begin{array}{l}\text { Protección } \\
\text { de ecosistemas } \\
\text { naturales. }\end{array}$ & $\begin{array}{l}\text { Franja costera del Delta } \\
12.738 \text { ha (terrestres) y } \\
35.647 \text { ha (marítimas). }\end{array}$ & $\begin{array}{l}\text { Parcialmente } \\
\text { (ambiental). }\end{array}$ \\
\hline $\begin{array}{l}\text { Carta Europea del } \\
\text { Turismo Sostenible. }\end{array}$ & 2007 & $\begin{array}{l}\text { Parque Natural } \\
\text { del Delta del Ebro } \\
\text { y Europarc. }\end{array}$ & $\begin{array}{l}\text { Turismo sostenible } \\
\text { y turismo de naturaleza. }\end{array}$ & $\begin{array}{l}320 \mathrm{~km}^{2} \\
\text { (toda la llanura deltaica). }\end{array}$ & Sí. \\
\hline $\begin{array}{l}\text { Destinos Europeos } \\
\text { de Excelencia (EDEN). }\end{array}$ & 2009 & $\begin{array}{l}\text { Parque Natural } \\
\text { del Delta del Ebro } \\
\text { y Comisión Europea. }\end{array}$ & $\begin{array}{l}\text { Turismo sostenible } \\
\text { y turismo de naturaleza. }\end{array}$ & $\begin{array}{l}320 \mathrm{~km}^{2} \\
\text { (toda la llanura deltaica). }\end{array}$ & Sí. \\
\hline $\begin{array}{l}\text { Catálogo del Paisaje } \\
\text { de las Tierras del Ebro. }\end{array}$ & 2010 & $\begin{array}{l}\text { Generalitat } \\
\text { de Catalunya. }\end{array}$ & Ordenación del paisaje. & $\begin{array}{l}320 \mathrm{~km}^{2} \text { (toda la llanura } \\
\text { deltaica) + comarcas } \\
\text { adyacentes. }\end{array}$ & Sí. \\
\hline $\begin{array}{l}\text { Plan Territorial de } \\
\text { las Tierras del Ebro. }\end{array}$ & 2010 & $\begin{array}{l}\text { Generalitat } \\
\text { de Catalunya. }\end{array}$ & Plan territorial general. & $\begin{array}{l}320 \mathrm{~km}^{2} \text { (toda la llanura } \\
\text { deltaica) + comarcas } \\
\text { adyacentes. }\end{array}$ & Sí. \\
\hline
\end{tabular}

Fuente: elaboración propia.

funcionamiento de ese territorio. Existe un estado de opinión creciente entre la mayoría de agentes del territorio favorable a impulsar políticas y actuaciones territoriales y socioeconómicas que favorezcan la sostenibilidad futura del delta, entendida ésta — tal como ya se ha dicho anteriormente- como el mantenimiento de un equilibrio entre las dinámicas biofísicas y socioeconómicas que se 
encuentran permanentemente en juego en el territorio deltaico. Este equilibrio debería garantizar la conservación de los ecosistemas naturales, al tiempo que la propia estabilidad física del delta, así como unas actividades económicas viables a largo plazo.

Sin embargo, es importante recordar que la sostenibilidad futura del delta va muy ligada también a las consecuencias del cambio climático a medio y largo plazo. Al ser la vulnerabilidad del delta muy alta en el largo plazo, y muy poco evidente en el corto, las medidas de adaptación al cambio climático han sido enfocadas prioritariamente en la franja litoral, la más sensible a los posibles impactos. En un primer momento del debate sobre esta cuestión, a principios de la primera década del siglo XXI, la prensa y las opiniones de los principales agentes territoriales del delta hablaron mucho (especialmente en contra) sobre las opciones defensivas enfocadas hacia una protección "dura» (con barreras físicas) del litoral. Las entrevistas realizadas por los autores en el marco del proyecto de investigación a los distintos grupos de agentes del territorio demostraron lo ajeno que era el tema de la adaptación al cambio climático para los habitantes, los agricultores, las instituciones y demás agentes. Las respuestas confundían adaptación y mitigación, y, principalmente, reclamaban protección para el delta, lo que significaba intentar mitigar los posibles impactos para que no afectaran al equilibrio actual del territorio (cosa imposible en el largo plazo). Mientras tanto, autores como Overeem y Syvitski (2009) exponían la preocupación de que, si no empezaba a desarrollarse inmediatamente ninguna medida de transformación y adaptación de los sistemas deltaicos amenazados, una hipótesis científicamente realística y cierta sería una destrucción de aquellos sistemas deltaicos en el largo plazo. Los mismos autores afirman que si, en el corto plazo, es sencillo actuar con medidas de protección, en el largo plazo, será muy costoso y casi inaceptable seguir con el mantenimiento de un equilibrio previo o una restauración del delta (Overeem y Syvitski, 2009).

En el año 2009, empezaron una serie de obras del nuevo Plan Integral de Protección del Delta del Ebro (PIPDE), que prevén una protección coherente de la franja litoral, entre ellas (Ministerio de Medio Ambiente, 2006):

a) La creación de un área de influencia, inicialmente propiedad privada y adquirida posteriormente por el Gobierno español y que pasa a ser área protegida.

b) La construcción de unas dunas artificiales (Barra de Trabucador y franja litoral de Sant Carles de la Ràpita) que hagan de barreras para posibles inundaciones y tormentas (que se prevé que aumentarán a corto o medio plazo) y permitan la renaturalización de estas áreas.

c) La construcción de un humedal artificial para la fitodepuración del agua de los riegos que desembocan en la bahía norte del delta, donde hay importantes zonas de producción acuícola.

Ese mismo año se dio a conocer la propuesta de construcción de diques o barreras artificiales que podrían estar financiados por el Plan Integral de Protección del Delta del Ebro, y que pretenden proteger, inicialmente, las 
dos bahías del delta y, a largo plazo, otras zonas costeras vulnerables del delta ante el cambio climático (Balsells, 2009). El primer dique previsto envolvería por el interior —a 100 metros de la línea de la costa — la bahía de Els Alfacs y tendría $14 \mathrm{~km}$ de longitud, 27 metros de ancho y hasta 1,2 metros de altura sobre el nivel del mar.

Hasta el momento presente, no nos consta ninguna obra, ni ningún proyecto, ni incluso debate, sobre estrategias de adaptación experimental a largo plazo que no entren en el esquema mental tradicional de protección del delta - basado en el corto plazo- , aunque existen distintas herramientas de gobernanza territorial, que permitirían desarrollar tales estrategias. Por ejemplo, el delta del Ebro ya cuenta con una cierta tradición de procesos participativos con los agentes del territorio, los cuales han permitido definir propuestas de estudios y actuaciones futuras para la zona. Sin embargo, cabe hacer una reflexión crítica sobre la filosofía de las recientes actuaciones de adaptación y protección del delta que si, en un primer momento, a corto plazo, son coherentes con los indicadores de vulnerabilidad de la zona, posteriormente pueden exacerbar las consecuencias de los posibles impactos futuros. $\mathrm{La}$ reflexión que queremos poner sobre la mesa trata de la relación entre resiliencia y sostenibilidad. De hecho, adaptaciones que, en el corto plazo, pueden parecer aliviar o solucionar un impacto (como la construcción de un dique), al mismo tiempo, reducen la percepción social del riesgo (de inundaciones, en el caso del dique) y aumentan la magnitud del posible daño futuro (en caso de una inundación más violenta en la que el dique no pueda resistirla). El caso de la evolución en la protección del territorio deltaico holandés explica muy claramente esta paradoja del "más defendido hoy, más vulnerable mañana», por la cual resiliencia y sostenibilidad no están necesariamente relacionadas. Cuando, en la década de 1970, se construían diques para la protección de las ciudades en los estuarios holandeses, en la de 1980, se reforzaban y, en la de 1990, continuaban creciendo los costes de protección, hasta que, a principios del nuevo milenio, se tuvo que optar por un cambio de paradigma, apostando más bien por una renaturalización de los mismos pólders para proteger mejor el sistema urbano. Eso significa que si, en una primera etapa, se intentaban proteger las viviendas, las zonas urbanas y la población de las inundaciones (siempre más frecuentes y fuertes), actualmente se intenta adecuar viviendas, zonas urbanas y hábitos ciudadanos a convivir con el agua, de manera que una inundación no represente un impacto peligroso, sino un factor ambiental que hay que aceptar en el funcionamiento del propio sistema (Schuetze y Chelleri, 2011). Esa clase de reflexión, de adaptación (resiliencia) mediante una transformación del mismo sistema (absorción del impacto en sus funciones) es la clave para llegar a la sostenibilidad de un sistema y representa el enlace entre resiliencia y sostenibilidad. Por su parte, la filosofía adaptativa (resiliencia en forma de resistencia) disminuye la exposición a los impactos y la percepción del riesgo a corto plazo, pero incrementa los costes de mantenimiento, así como la vulnerabilidad potencial a largo plazo, y, en definitiva, no garantiza la sostenibilidad del socioecosistema. 
En el caso del delta del Ebro, todas las intervenciones, como estudios sobre la vulnerabilidad del socioecosistema (por ejemplo: Taller d'Enginyeria Ambiental, 2008), intentan proteger el delta desde los principales impactos (subida del nivel del mar y posibles inundaciones) que podrían amenazar la zona con su configuración sistémica tal como la conocemos hoy en día, dependiente del (casi) monocultivo del arroz. Si bien coincidimos en que una protección del litoral, mediante proyectos de relevancia ambiental, como áreas protegidas y restauración de sistemas dunares (que fortalecen las dinámicas ecosistémicas de los humedales), constituyen una práctica positiva en términos generales, nos preguntamos si no sería necesario, al mismo tiempo, crear una plataforma de gobernanza que impulse un debate sobre las posibles transiciones y transformaciones que, a largo plazo, se puedan llegar a desarrollar en el delta desde un punto de vista sistémico socioecológico y, por lo tanto, también económico y cultural de uso del territorio, en sintonía con la filosofía seguida por el PIPDE.

Un problema evidente es que, actualmente, ese delta es muy dependiente de un equilibrio rígido y muy precario (un delta muy especializado en un monocultivo del arroz económicamente no rentable por sí mismo y financiado de momento, en buena parte, por subvenciones públicas) y, por lo tanto, poco resiliente, en el sentido de flexible a los cambios. Sin embargo, es preciso plantearse qué sucederá cuando próximamente entre en vigor la nueva política agraria comunitaria, que significará una progresiva disminución de las ayudas económicas al cultivo del arroz y podría llevar al abandono de la actividad en amplias zonas del delta por falta de viabilidad económica ante la entrada, en el mercado europeo, de arroz más barato proveniente de terceros países.

Teniendo en cuenta lo expuesto hasta el momento, parecería lógico que empezasen a debatirse opciones y estrategias de medio y largo plazo para que el sistema sea más elástico y pueda adaptarse (mientras se protege, de momento) a nuevas posibles situaciones ambientales, como un nivel más elevado de sal en el agua y el suelo, o inundaciones más frecuentes que puedan llegar a abarcar gran parte del territorio deltaico. Proyectos de investigación sobre cultivos resistentes a la sal, como proyectos piloto sobre transformación de los arrozales más cercanos a la franja costera hacia acuicultura o muchas otras posibles propuestas de diversificación funcional y económica de ese sistema deberían empezar a explorar sus posibles implementaciones a medio plazo para poder planear un delta resiliente y sostenible en el largo plazo, cuando están previstos los cambios y los impactos más notables en términos ambientales y territoriales. Hay suficientes evidencias y conocimiento científico como para saber que el delta no podrá seguir teniendo, de forma permanente en el tiempo, su estructura y sus usos actuales.

En relación con las alternativas al cultivo del arroz, cabe decir que son escasas y que se ven condicionadas por una especie de zonificación que coincide con los grandes dominios de vegetación potencial y que depende mayoritariamente de las cotas de nivel y de las características y la salinidad del suelo. En este sentido, el PIPDE propone algunas adaptaciones para tres zonas diferenciadas del delta (Ministerio de Medio Ambiente, 2006): 
a) Una primera zona caracterizada por las áreas de suelos salinos e hipersalinos que se inundan con facilidad debido a la topografía del terreno, que no supera la cota de $0,5 \mathrm{~m}$. El cultivo del arroz tiene aquí un rendimiento inferior al de otros lugares del delta, y su defensa contra los temporales marinos es costosa. Su extensión no es muy amplia y, por ello, se puede recomendar su recuperación como zonas húmedas naturales. Ello conlleva la posibilidad de otros usos, como, por ejemplo, la introducción de ganadería, como ya se ha realizado en el sector de La Tancada, o el acondicionamiento de zonas especializadas en acuicultura.

b) La segunda zona estaría constituida por suelos moderadamente salinos o con niveles freáticos hipersalinos próximos, con una cota que oscila entre los 0,5 y $1 \mathrm{~m}$ de altitud sobre el nivel del mar. El cultivo que más se adapta a esta zona es el arroz. Tanto por motivos ambientales como agrícolas, en esta zona no hay alternativa más viable que la del arroz. Su integración dentro del espacio protegido (Red Natura 2000 o Parque Natural) podría garantizar la obtención de ayudas económicas por motivos ambientales.

c) La última es la zona de mayor elevación topográfica con una cota superior a $1 \mathrm{~m}$. Son suelos muy poco salinos e inundados muy eventualmente. Es una superficie lo suficientemente ancha para poder plantear transformaciones de los cultivos actuales del arroz por huerta, tanto intensiva como extensiva con valor añadido.

\section{Conclusiones}

El delta del Ebro es un buen ejemplo de socioecosistema litoral sometido a un conjunto de presiones que amenazan su propia sostenibilidad a medio y largo plazo. En este artículo, se han explorado las relaciones entre la resiliencia y la sostenibilidad utilizando el delta del Ebro como caso de estudio para demostrar la diferencia entre adaptaciones en el corto plazo que llevan a la sostenibilidad o adaptaciones que llevan a la protección (disminución del nivel del riesgo), con un aumento progresivo de la vulnerabilidad sistémica. Se han analizado los planes y los instrumentos territoriales y ambientales vigentes actualmente en el delta del Ebro, constatando que los más recientes proponen y apuestan por un desarrollo sostenible y un delta teóricamente resiliente, aunque el concepto de resiliencia al cual se hace referencia no se delimita claramente en cuanto a transformación, transición o protección de sus elementos. Las evidencias de las actuaciones presentes muestran como se sigue apostando por el modelo de desarrollo actual (un delta dedicado casi exclusivamente al monocultivo de arroz), suavizando los impactos ambientales y climáticos del corto y medio plazos mediante la protección y la renaturalización de la franja litoral, medidas que, a largo plazo, debido al factor de incertidumbre, no son suficientes ni suficientemente enraizadas en la conciencia ni en el debate social. La protección ambiental del litoral, el control de la urbanización en todo el territorio deltaico y la monitorización ambiental son herramientas necesarias y fundamentales, siempre y cuando se desarrollen paralelamente otros instrumentos, como, por 
ejemplo, las innovadoras estrategias de transición (Hopkins, 2008; Grin et al., 2010), fundamentadas en una organización de la gobernanza y la construcción de conocimiento relevante para decidir en el largo plazo sobre las actividades que se verán afectadas por el cambio climático y otros retos socioambientales futuros.

El momento actual es importante y decisivo para el delta del Ebro, porqué es cuando se han empezado a desarrollar unas primeras medidas de adaptación y protección del delta ante los retos futuros, aunque no han dejado claro cuál es la filosofía de adaptación a medio y largo plazos, especialmente una vez el nivel del mar haya subido y las áreas inundables estén realmente inundadas (en el supuesto que las actividades del delta no hayan cambiado ni las áreas urbanizadas se hayan adecuado ni se haya restituido una aportación mínima de sedimentos al delta que permita compensar la regresión), momento en el cual el reto de la adaptación será aun más urgente. En consecuencia, se hace necesaria la implementación inminente (paralelamente a la protección y monitorización del delta) de otras herramientas de carácter más integrado - como la Gestión Integrada de Áreas Litorales_ y adaptativo — como la gobernanza adaptativa-, para poner sobre la mesa, con los actores locales, planificadores territoriales e investigadores de las ciencias naturales y sociales, escenarios futuros de desarrollo del delta, de sus actividades, configuración física y paisaje, teniendo en cuenta no sólo las actuaciones en el propio delta, sino también en toda la cuenca fluvial, ya que esas actuaciones, como ya se ha visto, acaban repercutiendo sobre la resiliencia del propio delta.

\section{Referencias bibliográficas}

Adger, N. (2000). «Social and ecological resilience: are they related?». Progress in Human Geography, 24, 347-364.

Agència Catalana De l'Aigua (2007). Pla de gestió del sediment del tram baix i final del riu Ebre i el seu delta (Pla GeSt Ebre). Barcelona. Generalitat de Catalunya.

Avallone, E.A.; BAumeIsTeR, T. y SADEGH, A.M. (2006). Marks'standard handbook for mechanical engineers. Nueva York: McGraw-Hill.

BALSELLS, F. (2009). «¿Salvará un muro el delta del Ebro?». El País, 27-07-2009.

BRETON, F. y SAURI-PUJOL, D. (1997). «Toward a redefinition of resources and hazards in coastal management: Examples from the lowland coastal areas of Catalonia, Spain». Coastal Management, 25, 363-385.

CARDOCH, L. y DAY, J.W. (2002). «Biophysical energy analyses of non-market values of the Ebro Delta". Journal of Coastal Conservation, 8, 87-96.

CurCO, A. (2006). «Aiguamolls litorals: El Delta de l'Ebre. Síntesi del medi físic d'una zona humida litoral». L'Atzavara, 14, 55-72.

Day, J.W.; MAltby, E. y IBÁÑ̂ZZ, C. (2006). «River basin management and delta sustainability: A commentary on the Ebro Delta and the Spanish National Hydrological Plan». Ecological Engineering, 26, 85-99.

Departament de Medi Ambient i Habitatge (2006). Proposta catalana a la Xarxa Natura 2000. Barcelona: Generalitat de Catalunya.

Departament de Política Territorial i Obres Públiques (2001). Pla Territorial de les Terres de l'Ebre. Barcelona: Generalitat de Catalunya. 
- (2009). Pla Territorial de les Terres de l'Ebre. Aprovació inicial. Juliol 2009. Barcelona: Generalitat de Catalunya.

EsCAlERA, J. y RuIZ, E. (2011). «Resiliencia socioecológica: Aportaciones y retos desde la antropología». Revista de Antropología Social, 20, 109-135.

European EnVIRONMENT AgEnCY (2006). The changing faces of Europe's coasts. Copenhague: EEA.

- (2010). Ecosystem Accounting for the Cost of Biodiversity Losses: Framework and Case Study for Coastal Mediterranean Wetlands. Copenhague: EEA.

FATORIC, S. y CHELlERI, L. (2012). «Vulnerability to the effects of climate change and adaptation: The case of the Spanish Ebro Delta». Ocean \& Coastal Management, $60,1-10$.

FOLKE, C. y BERKES, F. (1998). Linking social and ecological systems: management practices and social mechanisms for building resilience. Cambridge: Cambridge University Press.

Folke, C.; Carpenter, S.; Walker, B.; SCheffer, M.; Chapin, T. y Rockström, J. (2010). "Resilience thinking: integrating resilience, adaptability and transformability». Ecology and Society, 15 (4), 20.

Folke, C.; Hahn, T.; Olsson, P. y Norberg, J. (2005). «Adaptive governance of social-ecological systems». Annual Review of Environment and Resources, 30, 441-473.

Grin, J.; Rotmans, J. y SCHOT, J. (2010). Transitions to sustainable development: New directions in the study of long term transformative change. Nueva York: Routledge.

Guerrero, R.; Urmeneta, J. y RAmpone, G. (1993). «Distribution of types of microbial mats at the Ebro Delta, Spain». Biosystems, 31 (2-3), 135-144.

Holling, C.S. (1973). «Resilience and stability of ecological systems». Annual Review of Ecology and Systematics, 4, 1-23.

- (1996). «Engineering resilience versus ecological resilience». En: SCHUlzE, P.C. (ed.). Engineering within ecological constraints. Washington DC: National Academy of Engineering, 31-44.

Hopkins, R. (2008). The transition handbook: From oil dependency to local resilience. Foxhole: Green Books.

IBÁÑEZ, C.; CANICIO, A.; DAY, J.W. y CurCO, A. (1997). «Morphologic development, relative sea level rise and sustainable management of water and sediment in the Ebre Delta, Spain». Journal of Coastal Conservation, 3, 1-12.

Institut d'Estadística de Catalunya (2010). Anuari Estadistic de Catalunya 2010. Barcelona: Generalitat de Catalunya.

IPCC - Intergovernmental Panel for Climate Change (2001). Climate Change. Impacts, Adaptations and Vulnerability: Contribution to the Third Assessment report of the Intergovernmental Panel of Climate Change. Cambridge: Cambridge University Press, 641-693.

KAPLAN, H.B. (1999). "Toward an understanding of resilience: a critical review of definition and models». En: GLANTZ, M.D. y JOHNSON, J.L. (eds.). Resilience and development: positive life adaptation. Nueva York: Kluwer Academic/Plenum, 17-83.

LABIN, E.F. (2005). «Conditions for sustainability of human-environment systems: information, motivation, and capacity». Global Environmental Change, 15, 177-180.

LAGENDIJK, A. (2003). «Towards conceptual quality in regional studies: The need for subtle critique - a response to Markusen». Regional Studies, 37, 719-727.

Markusen, A. (1999). "Fuzzy concepts, scanty evidence, policy distance: the case for rigour and policy relevance in critical regional studies». Regional Studies, 37, 701-717. 
Mikhailova, M.V. (2003). "Transformation of the Ebro River Delta under the Impact of Intense Human-Induced Reduction of Sediment Runoff». Water Resources, 30 (4), 409-417.

Millennium ECOSYSTEM Assessment (2005). Ecosystems and human well-being: Wetlands and water. Synthesis. Washington, DC: World Resources Institute.

Miller, F.; Osbahr, H.; Boyd, E.; Thomalla, F.; Bharwani, S.; Ziervogel, G.; Walker, B.; Birkmann, J.; Van der LeeuW, S.; Rockström, J.; Hinkel, J.; Downing, J.; Folke, C. y Nelson, D. (2010). «Resilience and vulnerability: complementary or conflicting concepts?». Ecology and Society, 15 (3), art. 11. [http://www.ecologyandsociety.org/vol15/iss3/art11/]

Ministerio de Medio Ambiente (2006). Plan Integral para la Protección del Delta del Ebro. Documento base. Madrid: Ministerio de Medio Ambiente.

- (2007). Evaluación y conclusiones generales del ciclo de debate "El uso del agua en la Economía Española: situación y perspectivas». Madrid: Grupo de Análisis Económico / Fundación Biodiversidad.

Overeem, I. y Syvitski, J.P.M. (2009). Dynamics and Vulnerability of Delta Systems. LOICZ Reports \& Studies No. 35. Geesthacht: GKSS Research Center.

Parc Natural del Delta de l'Ebre (2005). El Parc Natural del Delta de l'Ebre. Barcelona: Generalitat de Catalunya.

- (2009). «El delta de l'Ebre i el Parc Natural, premi destinació europea d'excel.lència». Soldó. Informatiu del Parc Natural del Delta de l'Ebre, 33, 3.

REDMAN, C.L. y KIZING, A. (2003). «Resilience of past landscapes: resilience theory, society, and the longue durée». Conservation Ecology, 7 (1), 14.

Reid, W.V.; Chen, D.; Goldfarb, L.; Hackmann, H.; Lee, Y.T.; Mokhele, K.; Ostrom, E.; Raivio, K.; Rockström, J.; Schellnhuber, H.J. y Whyte, A. (2010). «Environment and development. Earth system science for global sustainability: grand challenges». Science, 330 (6006), 916-917.

RocKSTRÖM, J. et al. (2009). "A safe operating space for humanity». Nature, 461 (7263), 472-475.

Romagosa, F. (2000). Zones humides, societat $i$ medi ambient: Les zones humides de Catalunya. Bellaterra: Servei de Publicacions de la Universitat Autònoma de Barcelona.

Rovira, A. y IBÁÑEZ, C. (2007). «Sediment Management Options for the Lower Ebro River and its Delta». Journal of Soils and Sediments, 7 (5), 285-295.

SÁnCheZ-ArCilla, A.; JimÉnEZ, J.A. y Valdemoro, H.I. (1998). "The Ebro Delta: Morphodynamics and vulnerability». Journal of Coastal Research, 14, 754-772.

Scheffer, M.; Carpenter, S.; Foley, J.; Folke, C. y Walker, B. (2001). «Catastrophic shifts in ecosystems». Nature, 413 (6856), 591-596.

Schuetze, T. y Chelleri, L. (2011). "Climate adaptive urban planning and design with water in Dutch polders». Water Science and Technology, 64 (3), 722-730.

Slootweg, R. y VAn Beukering, P. (2002). Valuation of Ecosystem Services and Strategic Environmental Assessment. Lessons from Influential Cases. Utrecht: Netherlands Commission for Environmental Assessment.

TÀBARA, J.D.; ROCA, E. y MADRID, C. (2008). Developing new methods and tools for the integrated sustainability assessment of water. The MATISSE Project and the Ebro river basin. MATISSE Working Paper 8 . Viena: SERI.

TAller D'EngINYERIA AmBiental (2008). Estudis de base per a una estratègia de prevenció i d'adaptació al canvi climàtic N1: delta de l'Ebre. Document de sintesi. Barcelona. 
TURNER, B.L. (2010). «Vulnerability and resilience: coalescing or paralleling approaches for sustainability science?». Global Environmental Change, 20 (4), 570-576.

Valdemoro, H.I.; SÁnCHeZ-ArCilla, A. y JiménEZ, J.A. (2007). "Coastal dynamics and wetland stability. The Ebro delta case». Hydrobiologia, 577, 17-29.

Walker, B.; Holling, C.S.; Carpenter, S.R. y Kinzig, A. (2004). «Resilience, adaptability and transformability in social-ecological systems». Ecology and Society, $9(2), 5$.

WalKer, B.H. y SALt, D. (2006). Resilient thinking: Sustaining Ecosystems and people in a changing world. Washington, DC: Island Press.

YIN, R. (2003). Case study research: Design and methods. Thousand Oaks: Sage. 